\title{
The efficacy of self-directed learning versus problem-based learning for teaching and learning ophthalmology: a comparative study
}

This article was published in the following Dove Press journal:

Advances in Medical Education and Practice

\author{
Ihab Shafek Atta ${ }^{1,2}$ \\ Ali Hendi Alghamdi ${ }^{3}$ \\ 'Pathology Department, Faculty of \\ Medicine, Assuit Branch, Al-Azhar \\ University, Cairo, Egypt; ${ }^{2}$ Pathology \\ Department, Faculty of Medicine, \\ Albaha University, Albaha, Saudi \\ Arabia; ${ }^{3}$ Department of Surgery, \\ Faculty of Medicine, Albaha University, \\ Albaha, Saudi Arabia
}

Introduction: Self-directed learning (SDL) and problem-based learning (PBL) are fundamental tools to achieve lifelong learning in an integrated medical curriculum. However, the efficacy of SDL in some clinical courses is debated.

Aim: The aim of the study was to measure the effectiveness of SDL for an ophthalmology course in comparison with PBL.

Methods: A cross-sectional study was conducted with fifth-year medical students enrolled in an ophthalmology course. SDL comprised four case-based scenarios guided by several questions. PBL comprised three sessions. An ear, nose, and throat (ENT) course was selected for comparison as a control. At the end of the course, 30 multiple-choice questions (MCQs) for both SDL and PBL were assessed and analyzed against their counterparts in the ENT course by an independent $t$-test.

Results: For the SDL component of the ophthalmology course, the number and percentages of students attaining high $(n=6 / 60,10 \%)$ and moderate $(n=15 / 60,28.3 \%)$ scores on an MCQs written exam were evaluated. For the PBL component, high scores were seen for $23.3 \%(\mathrm{n}=$ $14 / 60)$, and moderate scores for $33.3 \%(n=20 / 60)$ of the participants. For the SDL component of the ENT course, the number and percentages of students attaining high $(n=14 / 60,23.3 \%)$ and moderate $(n=17 / 60,28.3 \%)$ scores were recorded. For the PBL component, high $(16 / 60$, $26.6 \%)$ and moderate $(17 / 60,28 \%)$ scores were recorded. Significant $p$-values were obtained between the results for SDL and PBL in the ophthalmology course $(p=0.009)$, as well as between SDL results for both courses $(p=0.0308)$. Moreover, differences between the SDL results of ophthalmology and the PBL results of ENT $(p=0.0372)$ were significant.

Conclusion: SDL appears to be less valuable for promotion of self-readiness. Periodic discussions in small groups or by panel discussion are strongly recommended for students to enhance readiness with SDL.

Keywords: SDL, PBL, ophthalmology, ENT, lifelong learning, teaching tools

\section{Introduction}

Self-directed learning (SDL) is an essential proficiency skill for medical practitioners and, as such, is a component of the medical curriculum at an early stage. Multiple modalities have been used for providing SDL instruction to undergraduate students, with numerous studies demonstrating the effectiveness of SDL as a means to increase student readiness and enthusiasm. ${ }^{1-3}$ The multiple SDL modalities found in various medical curricula are effective if the objectives are realistic and accomplishable, ensuring that learners can apply SDL modalities to situations wherein they are required to learn by themselves. ${ }^{4-6}$ One instructional SDL modality is the presentation of
Correspondence: Ihab Shafek Atta Pathology Department, Faculty of Medicine, (Assuit Branch), Al-Azhar University, Assiut, 71524, Egypt

Tel +201001556079

Email lhab.bassyouny@azhar.edu.eg 
case-based scenarios that guide learners by posing multiple problem-related questions. Those questions direct the learner to respond by using suggested learning resources. ${ }^{7-9}$

SDL is an efficient and effective learning tool for medical students. ${ }^{7}$ Several studies have demonstrated the value of SDL for learning physiology and anatomy. ${ }^{10,11}$ SDL facilitates self-governance as well as decision-making and communication skills. ${ }^{11,12}$ Numerous studies have compared SDL to traditional lectures, with some of these studies demonstrating that self-learning groups achieved better results than groups who received lectures. ${ }^{13-15}$ Other studies have found no difference between SDL and traditional classroom teaching. ${ }^{16-19}$

Problem-based learning (PBL) has been adopted by many medical schools, and those schools have used a number of diverse instructional approaches. The primary approach is the construction of case-based scenarios and problems for small groups of students who discuss the scenarios and derive accurate solutions to the problems. A tutor or facilitator offers sympathetic supervision of the students. Discussions are structured to permit student-generated theoretical approaches to clarify problems associated with the scenario or case. ${ }^{20}$ In this manner, students identify the limits of, or gaps in, their knowledge by recognition of the learning issues necessary to problem solution. Between individual group sessions, students investigate possible solutions to the problems. The students share their solutions and the results of their investigation at the next group session. ${ }^{21,22}$

PBL facilitates the student's acquisition of appropriate attitudes and skills. It enriches their development of communication skills, comprising cooperation, teamwork, and appreciation of the views of others, and promotes interaction with group members. ${ }^{21}$ Therefore, PBL is valuable for group education. ${ }^{23}$ Prince et $\mathrm{al}^{24}$ found that PBL enhanced attitudes and skills that promoted communication.

Barral and Buck ${ }^{20}$ described PBL as an educational practice that is widely used in many medical school curricula. Although there are many PBL variants, PBL practice implies the presentation of problems that are case based to a small group of students who discuss the problems for two or more sessions. A tutor advocates and provides sympathetic guidance for the students. The problems are devised in a manner that permits students to create theoretical models to solve the problems within the context of the presented case-based scenario.

PBL has become fashionable in many medical schools that have advocated for curriculum improvement by adopting an integration-based curriculum instead of a systembased one. An integration-based curriculum promotes SDL; encourages in-depth learning and thinking; prepares students for lifelong learning; and improves retention of knowledge, more so than traditional courses..$^{9,25}$

The medical school in the present study, Albaha School of Medicine (ABSM), adopted a fully integrated curriculum throughout the 6-year program, with the ophthalmology course implemented in the fifth clinical year. The learning objectives of the course were clearly defined and well structured, using taxonomy applied by Bloom and revised by Kress and Selander. ${ }^{5}$ The objectives were formulated by a modular committee composed of ophthalmology experts. The teaching strategies and tools were closely linked to the course objectives. SDL was implemented by students who selected subject areas or themes based on cases or scenarios identified within the study guide module.

The aim of the study was to assess the effectiveness of SDL for instruction of ophthalmology. Furthermore, a comparison was made of the impact of SDL and PBL on ophthalmology teaching, when implemented for medical students in the fifth year of the clinical phase at ABSM.

\section{Materials and methods Study design}

This cross-sectional study was approved by an ethical committee of ABSM, under the supervision of the Dean of Scientific Research and Quality as well as Accreditation Affairs. The committee approved and stated that "after investigation the proposal introduced by researchers to investigate the SDL vs. PBL in ophthalmology course, the committee agree to investigate the efficacy of SDL vs. PBL in the ophthalmology course as a part of a whole program evaluation without any funding" (ethical approval reference no. 211/2018). Furthermore, all students participating in the current study collectively provided written informed consent that implied their agreement to the research and investigation of a portion of their grades for SDL and PBL for both ophthalmology and the ear, nose, and throat (ENT) courses and to publish these data collectively in an anonymous form without individual detail.

At ABSM, the curriculum is a 6-year program. The academic years are divided into three phases: preparatory, basic, and clinical. The preparatory phase is the first academic year; the basic phase comprises the second and third years; and the clinical phase is the fourth, fifth, and sixth academic years. Each academic year has two levels (i.e. two semesters) arranged as follows: levels 1 and 2 forming the first academic year, levels 3 and 4 forming the second academic year, etc. The ophthalmology and ENT course are situated in the second 
semester of the fifth academic year, in Phase III, Level 9. In the preparatory phase, students study chemistry, biophysics, introductory courses for the basic sciences including pathology, innovation in medicine, and professionalism. In the basic phase, the students study a human body module, principle of disease, and systems-based basic modules or courses such as cardiovascular and respiratory. In the clinical phase, these courses are addressed and studied from a clinical perspective in addition to basic imaging, ophthalmology, ENT, and others. Each course is allocated credit hours and teaching strategy/ tools. Ophthalmology and ENT are allocated two credit hours each and the courses are implemented in a 2 -week period. The ophthalmology and ENT courses are in the second semester of the fifth academic year, in Phase III, Level 9 (Table 1).

This study was conducted with 60 male students enrolled in the ophthalmology course and representing the whole class of the fifth year, Phase III, and Level 9. At the time of the study, the students had completed the first five integrated modules adopted for that level, including the ENT course. All student grades for the ENT course were compared with the ophthalmology course for this study. The ENT course was selected as a control for the ophthalmology course in that the number of SDL and PBL is similar. Both courses are two credit units and of two weeks in duration, and are offered during the same year, level, and phase with the same number of questions posed for SDL/PBL.

The subjects for the study were selected and learning objectives designated for the ophthalmology course with four case-based scenarios adapted for both SDL and PBL. At the end of the SDL case scenario, guiding objectives were used to enable student identification of the differential diagnosis.

\section{Methods}

\section{Preparation of SDL and PBL material}

The content and learning objectives of the ophthalmology course were designed according to Bloom's taxonomy. The teaching strategy and tools were selected for each objective. Some content and objectives were selected for PBLs and others for SDL. In PBL, the selection criteria were dependent on the presence of more than one factor with regard to pathogenesis, risk factors, differential diagnoses, laboratory investigations, radiological investigations, and treatments. In

Table I Mapping of the ophthalmology and ENT courses within the curriculum

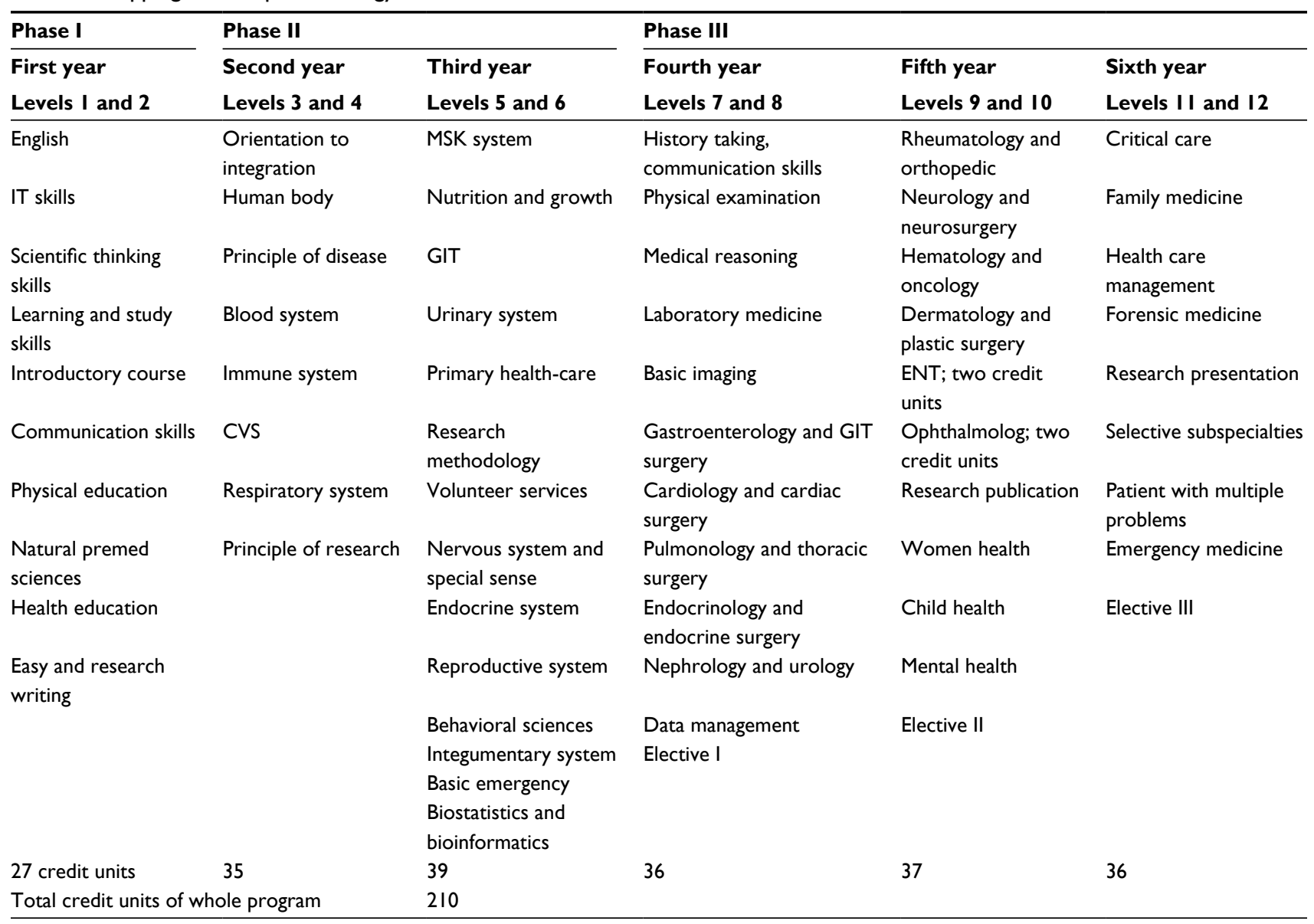

Abbreviations: CVS, cardiovascular system; ENT, ear, nose and throat; GIT, gastrointestinal; IT, information technology; MSK, musculoskeletal system; Premed, premedical. 
SDL, the criteria for selection were dependent upon genetic hypotheses and the rarity of the condition. These topics were not fully addressed by other teaching tools.

\section{For SDL}

The material for SDL was prepared by constructing two short case histories that covered the topic of ocular tumors. Each case was followed by learning objectives with the required references provided, which were derived from standard textbooks suggested at the beginning of the course. All 60 students completed SDL activities as a mandatory requirement for the course. The students read the scenario and discussed the learning objectives with the tutor and identified resources. Each group of ten students had a tutor who was a medical staff expert. The students investigated the case, undertook research, reported their findings, and discussed the case with their tutor, who provided continual guidance. At the conclusion of the course, a committee composed of staff experts discussed the case with each student separately, providing evaluation and feedback. A total of 15 questions related to the SDL activity were included in the final examination.

\section{For PBL}

The students were separated into five groups of 12 students each, with each group guided by a tutor. Two case-based scenarios were adopted. In the first session, the students discussed the case and addressed the learning objectives under the supervision of a tutor. In the second session, the students discussed their findings on case management and differential diagnosis. In the third session, all student groups were brought together as a single class. The tutors for all groups formed a committee that selected students (at least one student from each group) to deliver findings for each objective. This last session ensured that all learning objectives were delivered to all students in an equivalent manner by the end of the course. A total of 15 questions representing the PBL activity were included in the final examination.

The tutors selected for both SDL and PBL were staff medical experts from different departments. The tutors were trained by the medical education department through several workshops that specified the conduct of successful SDL and PBL as well as a description of the role of the tutor.

An example of PBL and learning objectives for the ophthalmology course are presented in Box 1.
Box I Example of PBL and learning objectives for the ophthalmology course

\begin{abstract}
A 35-year-old convenience store manager with a history of noninsulin-dependent DM for 5 years presents to the Eye Clinic for his annual visit. He admits to a progressive blurring of vision since his last visit a I year ago. Reading street signs while driving seems more difficult. He is unsure of which eye seems worse. He does not wear contacts and uses over-the-counter reading glasses. He denies any flashes or floaters, diplopia, eye discomfort, or pain. The patient reports that he has been going through some tough times recently, with a difficult divorce approximately 6 months earlier. As a result of all the stress, his blood sugars have not been under good control and he feels he has been making this worse by eating a lot of junk food and not always taking his diabetic medications. His blood sugar has been as high as 400 and he was admitted to the hospital 3 months back for diabetic ketoacidosis. Past ocular history: No prior eye surgeries, no history of eye trauma, amblyopia, or strabismus. No prior diabetes findings in the eye. Ocular medications: None. Medical history: Hypercholesterolemia, obesity, DM Type 2, and hypertension. Surgical history: None. Family ocular history: Negative for macular degeneration, glaucoma, diabetic retinopathy, or blindness. Social history: 30 pack-year smoking history and drinks alcohol socially. Medications: Lisinopril, hydrochlorothiazide, metformin, and simvastatin. Allergies: None. Other systems: Normal. Ocular examination: Visual acuity: OD: 20/40, OS: 20/40. Intraocular pressure: OD: $16 \mathrm{mmHg}$; OS: $15 \mathrm{mmHg}$. Pupils: Equal, round, and reactive to light. No APD. Extraocular movements: Full OU. No nystagmus. Confrontational visual fields: Full to finger counting $O U$. External: Normal, both sides. Slit lamp examination: Normal. Dilated fundus examination: OD: Clear view, CDR 0.35; neovascularization of the disc involving $~ 50 \%$ of the disc; flat macula with multiple microaneurysms and hard exudates $>500$ microns away from the fovea, no clinically significant macular edema; multiple dot-blot hemorrhages in the retinal periphery in all four quadrants without retinal detachment. OS: Clear view, CDR 0.40 with sharp optic disc margins; flat macula with multiple microaneurysms and hard exudates $>500$ microns away from the fovea; no clinically significant macular edema; peripheral retina with multiple dot-blot hemorrhages in the periphery in all four quadrants.
\end{abstract}

\section{Objectives: at the end of PBL sessions, all students will be} able to:

I. Define diabetic retinopathy (DR)

2. Identify the pathogenesis, CP, classification, risk factors, and management of DR

3. Perform visual rehabilitation of patients with advanced DR.

Abbreviations: $A P D$, afferent pupillary defect; $C D R$, cup disc ratio; $C P$, clinical picture; DM, diabetes mellitus; OD, right eye; OS, left eye; OU, both eyes; PBL, problem-based learning; Premed, premedical.

An example of SDL and learning objectives for the ophthalmology course are presented in Box 2.

An example of PBL and learning objectives for the ENT course are presented in Box 3.

An example of SDL and learning objectives for the ENT course are presented in Box 4. 
Box 2 Example of SDL and learning objectives for the ophthalmology course

SDL: Ocular tumors
Short-case scenario: A 45 -year-old male patient presented with
leukocoria plus DV and unilateral eye protrusion in his left eye. OCT
revealed an ocular mass. How to manage this case?
At the end of this SDL, the students will be able to:
I. Identify eye tumors such as retinoblastoma, malignant melanoma,
optic nerve glioma, and optic nerve sheath meningioma
2. Outline the management of a case with ocular tumor.
The most common ocular tumor presenting early with
leukocoria is:
a) Retinoblastoma
b) Malignant melanoma
c) Optic nerve glioma
d) Optic nerve sheath meningioma.

Abbreviations: DV, defective vision; OCT, ocular computerized tomography; SDL, self-directed learning.

Box 3 Example of PBL and learning objectives for the ENT course

Mohamed is a 30-year-old male from Al-Aqiq. Two years ago,
he presented to Al-Aqiq Hospital suffering from nasal itching,
sneezing, and mild fever. Dr Nowaf examined him and found that
his nose appeared pale, the nasal mucosa was congested, and
inferior turbinates were enlarged. Otoscopic examination revealed
red, bulging tympanic membrane in the left ear. Based on these
findings, Dr Nowaf prescribed an oral antibiotic, antipyretic, and
nasal decongestant. One month following his first visit, Mohamed
returned with scanty, offensive, mucopurulent discharge of the left
ear. The discharge was associated with severe ear pain, hearing
loss, tinnitus, and vertigo. On examination, there was a visible large
posterior perforation in the tympanic membrane at the attic area with
edematous mucosa of the middle ear, granulation tissue, and aural
polyp protruding through the perforation. The doctor conducted
good aural toilet and requested a computed tomography (CT) scan
of the left mastoid bone. The CT showed a cavity within the mastoid.
Two weeks later, Mohamed underwent surgery of his left ear and
mastoid bone where some tissues were removed and another tissue
was added. Mohammed felt good, and his hearing was restored.
At the end of the PBL session, the students will be able to:
I. Revise the anatomy and histology of the middle ear
2. Identify the etiology, clinical picture, and investigations required
4. Design the management plan for chronic and acute otitis media
5. Be aware of the risk factors, epidemiology, prevention, and control
of acute and chronic otitis media.
An example of question applied
Which of the following diagnostic techniques is the most
essential for otitis media?
a) Pneumatic otoscopy
b) Tympanometry
c) Tympanocentesis
d) Acoustic reflectometry.

Abbreviations: ENT, ear, nose and throat; PBL, problem-based learning.
Box 4 An example of self directed learning (SDL) with question applied for ENT course

\section{SDL: Neck mass}

Case scenario: A 36-year-old female patient presented with a neck mass. The patient had a history of neck swelling since birth that remained dormant up to the previous 2 months during which the patient noticed the swelling was increasing.

At the end of SDL, the students will be able to:

I. Describe the approach to a neck mass

2. List different types of congenital neck masses

3. Describe the management of congenital neck masses

4. Categorize the types of premalignant lesions and their treatment

5. List different types of head and neck malignancies and their management

6. Identify the different types of thyroid carcinoma and their management.

Which one of the following thyroid tumors is considered a part of MEN syndrome?
a) Papillary
b) Follicular
c) Medullary
d) Anaplastic

Abbreviations: MEN, multiple endocrinal neoplasia; SDL, self-directed learning.

\section{Assessment and statistical analysis}

Thirty questions representing SDLs and PBLs were included in the final examination with 15 questions for each. The students answered these questions, and results were recorded with no negative marks applied. Comparisons were done by an independent sample $t$-test. For each tool, the student scores (maximum of 15) were categorized into high (score $\geq 13$ ), moderate (score 11-12), low (score $=9,10$ ), and very low (score $<9$ ). A $p$-value of $<0.05$ was considered significant. SPSS for Windows version 17.0 (SPSS Inc., Chicago, IL, USA) was utilized for data analysis.

\section{Results}

The answers for the 30 questions, 15 each for SDL and PBL, for the 60 students were as follows: With regard to the SDL component of the ophthalmology course, the number of students and the percentages were as follows: high scores in $10 \%(\mathrm{n}=6 / 60)$, moderate in $28.3 \%(\mathrm{n}=15 / 60)$, low in $38.3 \%(23 / 60)$, and very low in $26.6 \%(\mathrm{n}=16 / 60)$. For PBL, we observed high scores in $23.3 \%(\mathrm{n}=14 / 60)$, moderate in $33.3 \%(n=20 / 60)$, low in $25 \%(15 / 60)$, and very low in $18.3 \%(n=11 / 60)$ (Table 2). For the SDL component of the ENT course, the number and percentages were: high scores in $23.3 \%(n=14 / 60)$, moderate in $28.3 \%(n=17 / 60)$, low in $26.6 \%(n=16 / 60)$, and very low in $21.6 \%(n=13 / 60)$. For 
Table 2 Number and percentages of each group for SDL and PBL for both ophthalmology and ENT courses with statistical analysis by independent $t$-test

\begin{tabular}{lllllll}
\hline Course & Tool & High, $\mathbf{n}(\%)$ & Moderate, $\mathbf{n}(\%)$ & Low, $\mathbf{n}(\%)$ & Very low, $\mathbf{n}(\%)$ & t-test \\
\hline Ophth & SDL & $6(10)$ & $15(25)$ & $23(38.3)$ & $16(26.6)$ & $p$-value of 0.00941 is \\
& PBL & $14(23.3)$ & $20(33.3)$ & $15(25)$ & $11(18.3)$ & highly significant \\
ENT & SDL & $14(23.3)$ & $17(28.3)$ & $16(26.6)$ & $13(21.6)$ & $p$-value of 0.5 not \\
& PBL & $16(26.6)$ & $17(28.3)$ & $10(16.6)$ & $17(28.3)$ & significant \\
\hline
\end{tabular}

Notes: $p$-value using $t$-test. Significant $p$-value between the two SDLs $(p=0.0308)$, significance obtained between SDL of ophthalmology and PBL of ENT $(p=0.03724)$. No significance was obtained between PBL of both courses nor between SDL of ENT and PBL of the ophthalmology course, with $p=0.3406$ and 0.3340 , respectively.

Abbreviations: ENT, ear nose and throat; Ophth, ophthalmology; PBL, problem-based learning; SDL, self-directed learning.

PBL, we observed high scores in $26.6 \%(\mathrm{n}=16 / 60)$, moderate in $28.3 \%(\mathrm{n}=17 / 60)$, low in $16.6 \%(10 / 60)$, and very low in $28.3 \%(\mathrm{n}=17 / 60)$ (Table 2 and Figure 1). A significant difference between SDL and PBL for the ophthalmology course was observed $(p=0.0094)$. Significant differences were observed between the two SDLs $(p=0.0308)$ and between the SDL of ophthalmology and the PBL of ENT $(p=0.03724)$ (Table 2). Further analysis of SDL and PBL against the total scores of students in the ophthalmology course demonstrated significant differences between students attaining 80-89\% in SDL and PBL, between 70-79\% for both SDL and PBL with $p$ value $=0.0196$, and 0.01189 , respectively (Table 3 ).

\section{Discussion}

SDL has become a fundamental instructional modality for adult learning. In the health profession, SDL skills are linked to lifelong learning, ${ }^{26}$ with such learning central to understanding advances in medical knowledge and improved innovations in patient care. ${ }^{26}$

Based on data in Table 2, students enrolled in the SDL component of the ophthalmology course were deficient relative to those enrolled in PBL in the same course as well as its counterpart in the ENT course. A possible explanation is that students may consider the small number of SDL examination questions and the short duration of the course insufficient for adequate consideration and reading. These results are similar to those reported from a study by Murphy et $\mathrm{al}^{27}$ who found that SDL is not an appropriate learning method for anatomy. Those authors found that student's recall knowledge was derived from didactic lectures and not from reading about the subject.

Similar to the results herein, Pai et $\mathrm{al}^{28}$ evaluated the effectiveness of SDL for first-year medical students divided into two groups - one that received lecture plus SDL on the same topic and another group that received lecture only. Ten multiple-choice questions evaluated whether there was a difference between the two groups for physiology learning, and no difference was found.

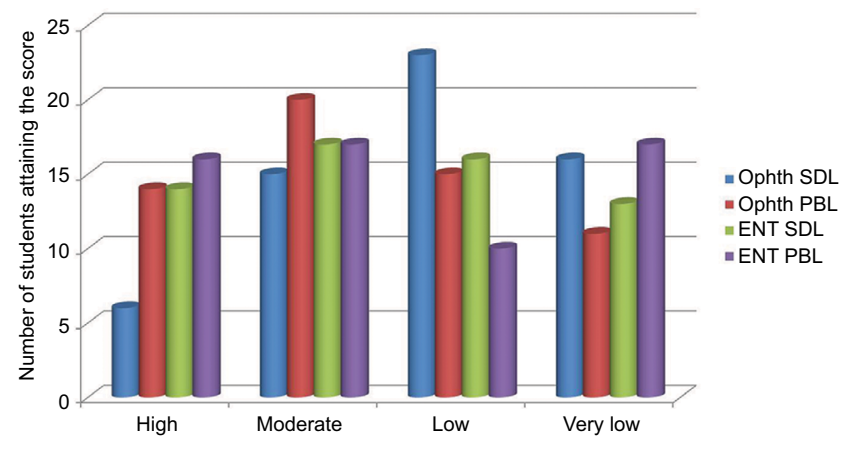

Figure I Student SDL and PBL scores for both the ophthalmology and ENT courses. Abbreviations: ENT, ear nose and throat; Ophth, ophthalmology; PBL, problembased learning; SDL, self-directed learning.

The PBL results of the ophthalmology course demonstrate the importance of PBL within the integrated curriculum - in particular, the ophthalmology course. These results are similar to many studies indicating the important role of PBL in adult learning and enhanced student performance. ${ }^{9,29}$

The results for SDL and other elements indicate that the delivery of SDL is problematic and needs reform. With regard to PBL, no significant difference between the ophthalmology and ENT courses was observed, with good student achievement in both courses. This identifies a gap between the SDL components of the two courses.

In the present study, analysis of ophthalmology scores for SDL and PBL revealed a significant $p$-value between the $80 \%-90 \%$ group $(p=0.0196)$ and the $70 \%-79 \%$ group ( $p=0.01189$ ) (Table 3$)$. These results suggest that the weakest SDL achievement was observed in the majority of students. Further, in the high-scoring group, two students showed low and very low scores for SDL. This may be due to inappropriate SDL management in which student needs were not identified, learning objectives were not understood, or motivation was lacking for those students. Overall, these data demonstrate the need for SDL reform by the committee. 
Table 3 Distribution of students according to their grades in SDL and PBL based on their total score in the ophthalmology course

\begin{tabular}{|c|c|c|c|c|c|c|}
\hline Score & Tool & High & Moderate & Low & Very low & $\begin{array}{l}p \text {-value using } \\
\text { independent } t \text {-test }\end{array}$ \\
\hline \multirow[t]{2}{*}{$\geq 90$} & SDL & 2 & 3 & 1 & 1 & $0.407 I$ \\
\hline & PBL & 3 & 2 & I & I & \\
\hline \multirow[t]{2}{*}{$80-89$} & SDL & I & 3 & 6 & 5 & $0.0196^{*}$ \\
\hline & PBL & 7 & 2 & 3 & 3 & \\
\hline \multirow[t]{2}{*}{ 70-79 } & SDL & I & 4 & 11 & 6 & $0.01189 *$ \\
\hline & PBL & 2 & 12 & 5 & 3 & \\
\hline \multirow[t]{2}{*}{$60-69$} & SDL & I & 2 & 3 & 2 & 0.3209 \\
\hline & PBL & 1 & I & 3 & 3 & \\
\hline \multirow[t]{2}{*}{$<60$} & SDL & I & 3 & 2 & 2 & 0.4026 \\
\hline & PBL & I & 3 & 3 & I & \\
\hline
\end{tabular}

Note: *There are significant differences in the student group attaining score of $80-89 \%$ for SDL and PBL, and also in-between the student group attaining $70-79 \%$ for SDL and PBL with $p=0.019$ and 0.011 , respectively.

Abbreviations: PBL, problem-based learning; SDL, self-directed learning.

Blumberg ${ }^{30}$ studied the effect of PBL on SDL and found that student involvement in PBL improved utilization of SDL skills. Some medical schools have identified particular courses within their curricula that are based on SDL activities in order to cultivate lifelong learning. Another study recommended that a primary goal for faculty is to encourage SDL among students in order to promote students' lifelong learning and to enhance their skills." ${ }^{31}$

Shokar et $\mathrm{al}^{32}$ assessed the degree of readiness for SDL in third-year medical students who participated in a PBL curriculum during the first 2 years of medical school. Students in this integrated medical curriculum were found to have good reading achievement through SDL that correlated with clinical performance. Those authors concluded that higher student achievement and readiness were achieved with SDL, which was associated with clinical clerkship scores and improved clinical skills. Others recommended that SDL be imbedded into the medical curriculum by formulating well-structured and staged courses. ${ }^{33}$

Grow $^{33}$ advocated that SDL skills be purposefully integrated into the curriculum through a staged planning model. ${ }^{16}$ Although SDL is strongly recommended for integration-based systems, Candy advised implementation in a systems-based approach. ${ }^{26}$ It is worth noting that learners who have a deficiency in SDL experience are unable to master SDL skills or provide that experience for their stakeholders. ${ }^{34}$

Concerning SDL, many suggestions have been made to improve the efficacy and effectiveness of teaching strategies such as SDL. These suggestions include clarification of learning objectives, periodic tutor driven discussions with students, the formation of small groups, panel discussions with the students about SDL themes, and identification of student learning styles. ${ }^{35-37}$ These suggestions have been implemented in courses with good results.

\section{Conclusion}

SDL is important for lifelong learning, especially in an integration-based curriculum. However, for this ophthalmology module, SDL did not promote self-readiness. Recommendations for improvement include instruction of more periodic small groups and panel discussions with the students.

\section{Disclosure}

The authors report no conflicts of interest in this work.

\section{References}

1. Bergman EM, Sieben JM, Smailbegovic I, de Bruin AB, Scherpbier AJ, van der Vleuten CP. Constructive, collaborative, contextual, and self-directed learning in surface anatomy education. Anat Sci Educ. 2013;6(2):114-124.

2. Lee YM, Mann KV, Frank BW. What drives students' self-directed learning in a hybrid PBL curriculum? Adv Health Sci Educ Theory Pract. 2010;15(3):425-437.

3. Premkumar K, Pahwa P, Banerjee A, Baptiste K, Bhatt H, Lim HJ. Does medical training promote or deter self-directed learning? A longitudinal mixed-methods study. Acad Med. 2013;88(11):1754-1764.

4. Ainoda N, Onishi H, Yasuda Y. Definitions and goals of "self-directed learning" in contemporary medical education literature. Ann Acad Med Singapore. 2005;34(8):515-519.

5. Kress G, Selander S. Multimodal design, learning and cultures of recognition. Internet High Educ. 2012;15(4):265-268.

6. Findlater GS, Kristmundsdottir F, Parson SH, Gillingwater TH. Development of a supported self-directed learning approach for anatomy education. Anat Sci Educ. 2012;5(2):114-121.

7. Harvey BJ, Rothman AI, Frecker RC. Effect of an undergraduate medical curriculum on students' self-directed learning. Acad Med. 2003;78(12):1259-1265.

8. Lycke KH, Grøttum P, Strømsø HI. Student learning strategies, mental models and learning outcomes in problem-based and traditional curricula in medicine. Med Teach. 2006;28(8):717-722. 
9. Atta IS, AlQahtani FN. Hybrid PBL radiology module in an integrated medical curriculum Al-Baha Faculty of Medicine experience. J Contemp Med Educ. 2015;3(1):46-51.

10. Arroyo-Jimenez Mdel M, Marcos P, Martinez-Marcos A, et al. Gross anatomy dissections and self-directed learning in medicine. Clin Anat. 2005;18(5):385-391.

11. Grieve C. Knowledge increment assessed for three methodologies of teaching physiology. Med Teach. 1992;14(1):27-32.

12. Lake DA. Student performance and perceptions of a lecture-based course compared with the same course utilizing group discussion. Phys Ther. 2001;81(3):896-902.

13. Abraham RR, Upadhya S, Ramnarayan K. Self-directed learning. $A d v$ Physiol Educ. 2005;29(2):135-136.

14. Abraham RR, Fisher M, Kamath A, Izzati TA, Nabila S, Atikah NN. Exploring first-year undergraduate medical students' selfdirected learning readiness to physiology. Adv Physiol Educ. 2011;35(4):393-395.

15. Gade S, Chari S. Case-based learning in endocrine physiology: an approach toward self-directed learning and the development of soft skills in medical students. Adv Physiol Educ. 2013;37(4):356-360.

16. Peng WW. Self-directed learning: a matched control trial. Teach Learn Med. 1989;1:78-81.

17. Haidet P, Morgan RO, O’Malley K, Moran BJ, Richards BF. A controlled trial of active versus passive learning strategies in a large group setting. Adv Health Sci Educ Theory Pract. 2004;9(1):15-27.

18. Bradley P, Oterholt C, Herrin J, Nordheim L, Bjørndal A. Comparison of directed and self-directed learning in evidence-based medicine: a randomised controlled trial. Med Educ. 2005;39(10):1027-1035.

19. Finley JP, Sharratt GP, Nanton MA, Chen RP, Roy DL, Paterson G. Auscultation of the heart: a trial of classroom teaching versus computerbased independent learning. Med Educ. 1998;32(4):357-361.

20. Barral JM, Buck E. What, how and why is problem-based learning in medical education? Annu Rev Biochem. 2013;12(8):34-35.

21. Wood DF. ABC of learning and teaching in medicine: problem based learning. BMJ. 2003;326(7384):328-330.

22. Onyon C. Problem-based learning: a review of the educational and psychological theory. Clin Teach. 2012;9(1):22-26.

23. Dolmans DH, De Grave W, Wolfhagen IH, van der Vleuten CP. Problembased learning: future challenges for educational practice and research. Med Educ. 2005;39(7):732-741.
24. Prince KJ, van Eijs PW, Boshuizen HP, van der Vleuten CP, Scherpbier AJ. General competencies of problem-based learning (PBL) and nonPBL graduates. Med Educ. 2005;39(4):394-401.

25. Kilroy DA. Problem based learning. Emerg Med J. 2004;21(4):411-413.

26. Candy PC. Self-Direction for Lifelong Learning: a Comprehensive Guide to Theory and Practice. San Francisco, CA: Jossey-Bass; 1991.

27. Murphy KP, Crush L, O’Malley E, et al. Medical student knowledge regarding radiology before and after a radiological anatomy module: implications for vertical integration and self-directed learning. Insights Imaging. 2014;5(5):629-634.

28. Pai KM, Rao KR, Punja D, Kamath A. The effectiveness of self-directed learning (SDL) for teaching physiology to first-year medical students. Australas Med J. 2014;7(11):448-453.

29. Chang BJ Problem-based learning in medical school: a student's perspective. Ann Med Surg (Lond). 2016;12:88-89. eCollection 2016.

30. Blumberg P. Evaluating the evidence that problem-based learners are self-directed learners: a review of the literature. In: Evensen DH, Hmelo CE, editors. Problem-Based Learning: a Research Perspective on Learning Interactions. Mahwah, NJ: Lawrence Erlbaum Associates; 2000:199-226.

31. Swanson AG, Anderson MB. Educating medical students. Assessing change in medical education - the road to implementation. Acad Med. 1993;68(6 Suppl):S1-S46.

32. Shokar GS, Shokar NK, Romero CM, Bulik, RJ. Self-directed learning: looking at outcomes with medical students. Fam Med. 2002;34(3): 197-200.

33. Grow G. Teaching learners to be self-directed. Adult Educ $Q$. $1991 ; 41: 125-149$

34. Diaz RM, Berk LE. A Vygotskian critique of self-instructional training. Develop Psychopathol. 1995;7(2):369-392.

35. Atta IS, AlQahtani FN. How to adjust the strategy of radiopathologic teaching to achieve the learning outcomes? Int J Med Sci Public Health. 2018;7(2):86-91

36. Atta IS, AlQahtani FN. Matching medical student achievement to learning objectives and outcomes: a paradigm shift for an implemented teaching module. Adv Med Educ Pract. 2018;9:227-233.

37. Atta IS, AlQahtani FN, Alghamdi TA, Mankrawi SA, Alamri AM. Can Pathology - teaching' strategy be affected by the students' learning style and to what extent the students' performance be affected? Glo Adv Res J Med Med Sci. 2017;6(11):296-301.
Advances in Medical Education and Practice

\section{Publish your work in this journal}

Advances in Medical Education and Practice is an international, peerreviewed, open access journal that aims to present and publish research on Medical Education covering medical, dental, nursing and allied health care professional education. The journal covers undergraduate education, postgraduate training and continuing medical education
Dovepress

including emerging trends and innovative models linking education, research, and health care services. The manuscript management system is completely online and includes a very quick and fair peer-review system. Visit http://www.dovepress.com/testimonials.php to read rea quotes from published authors. 